Hirosaki Med. J. 61(Suppl.) : S142-S146, 2010

\title{
NOVEL RAT MIDDLE CEREBRAL ARTERY OCCLUSION MODEL: TRANS- FEMORAL ARTERY APPROACH COMBINED WITH PRESERVATION OF THE EXTERNAL CAROTID ARTERY
}

\author{
Norihito Shimamura M.D., Ph.D. and Hiroki Ohkuma M.D., Ph.D.
}

\begin{abstract}
We developed a novel trans-femoral artery approach to the rat middle cerebral artery occlusion model (TF-MCAO) without sacrificing the external carotid artery (ECA) with/without the pterygopalatine artery, which is important for chewing food. To make the TF-MCAO we first dissect the left common carotid artery (CCA), ECA, and internal carotid artery (ICA). Transient occlusion clips are applied to the proximal ECA and the pterygopalatine artery; we never sacrifice the ECA branch. A 24-gage catheter is inserted into the left femoral artery. We insert a slightly bent 0.014 inch hydrophilically-coated guide wire via a haemostasis valve. Anatomically, the left common carotid artery is located rostral to the descending aorta, permitting a straight-forward, blind approach to the CCA. The guide wire is gently advanced about $17 \mathrm{~mm}$ from the bifurcation of the CCA until slight resistance is encountered. The guide wire and temporary occlusion clips are withdrawn after 90 minutes. Rats were sacrificed 24 $\mathrm{h}$ after reperfusion. Eleven rats were examined. One rat died before occlusion due to deep anesthesia. The success rate for producing infarction was $80 \%$. The mean infarction volume of the basal ganglia was $94.4 \mathrm{~mm}^{3} \pm 9.4 \mathrm{se}$ and mean infarction volume of the cerebral cortex was $124.2 \mathrm{~mm}^{3} \pm 21.6$ se. No rat died due to cerebral infarction and no rat suffered subarachnoid hemorrhage. We conclude that TF-MCAO was useful for producing a cerebral infarction.
\end{abstract}

Hirosaki Med. J. 61, Supplement : S142-S146, 2010

Key words: Cerebral ischemia; micro guide wire; neurological evaluation; rat; trans-femoral artery approach.

\section{Introduction}

The rat intraluminal suture method of middle cerebral artery occlusion (MCAO) was first reported by Koizumi et al. in $1986^{1)}$. Following that, a large number of reports on the rat intraluminal suture MCAO model has been published ${ }^{2-5}$. All of those methods must sacrifice the external carotid artery (ECA) with or without sacrificing the pterygopalatine artery. Sacrificing the ECA induces ischemic tissue damage of the arterial territory, causing impaired mastication and swallowing ${ }^{6}$.

We previously reported that commercially available silicon coated 4-0 nylon suture was suitable to produce rat $\mathrm{MCAO}^{7}$. But we had also sacrificed the ECA and pterygopalatine artery to make the rat MCAO model. If we can instead preserve all branches of the ECA, nutrition of $\mathrm{MCAO}$ rats improves and long term survival study projects that require a therapeutic diet can be carried out.

We have now created a novel rat MCAO model via a trans-femoral artery approach (TFMCAO). This model preserves the ECA and pterygopalatine artery. In this paper we evaluate the steady result of the infarction volume with a 0.014 inch hydrophilic occlusion guide wire and correlate the neurological evaluation and the infarction volume.
Department of Neurosurgery, Hirosaki University School of Medicine

Corresponding author: Norihito Shimamura M.D., Ph.D.

Department of Neurosurgery
Hirosaki University School of Medicine 5-Zaihuchou, Hirosaki Aomori pref. JAPAN. 036-8562

Tel: +81-172-39-5115 Fax : +81-172-39-5116

e-mail: shimab@cc.hirosaki-u.ac.jp 


\section{Material and Methods}

All experiments were approved by the Hirosaki University Animal Care Committee and conformed to the guidelines provided by the American Academy of Accreditation of Laboratory Animal Care.

Eleven male Sprague-Dawley rats weighing 290 to $310 \mathrm{~g}$ were housed under identical, controlled $12 \mathrm{~h}$ light-cycling conditions. The rats had free access to water and food and were anesthetized with $0.4 \mathrm{mg} / 100 \mathrm{~g}$ body weight (BW) intra-peritoneal (ip) atropine sulfate hydrate (Mitsubishi Tanabe Pharma Co., Osaka, Japan), $1 \mathrm{mg} / 100 \mathrm{~g} \mathrm{BW}$ ip xylazine (Bayer Schering Pharma, Osaka, Japan) and $8 \mathrm{mg} / 100 \mathrm{~g}$ BW ip ketamine hydrochloride (Daiichi Sankyo Co., Tokyo, Japan).

\section{MCAO model}

After general anesthesia rats were placed in the supine position on the operating table (Fig. 1A). Rectal temperature of rats was kept at $37 \pm 1{ }^{\circ} \mathrm{C}$ from the start of anesthesia to awakening. Initially, we exposed the left common carotid artery (CCA), ECA, and the internal carotid artery (ICA) through a midline incision. Temporary occlusion clips were applied to the proximal ECA and the pterygopalatine artery; we never sacrificed the ECA branch (Fig. 1B).

Next, a left femoral $1 \mathrm{~cm}$ skin incision was performed and a 24-gage catheter (Angiocath ${ }^{\circledR}$, Becton Dickinson Co., Fukushima Japan, $0.7 \mathrm{x}$ $1.9 \mathrm{~mm}$ ) was inserted into the left femoral artery under a microscope (Fig. 1C). We inserted a slightly bent 0.014 inch hydrophilically-coated guide wire (Transend EX Platinum ${ }^{\circledR}$, Boston Scientific, Fairmont, CA, USA) via a haemostasis valve (Radifocus ${ }^{\circledR}$ Haemostasis Valve II, Terumo, Tokyo, Japan) (Fig. 2). Anatomically, the left common carotid artery is located rostral to the descending aorta, permitting a straightforward, blind approach to the CCA without high resolution fluoroscopy. The guide wire was gently advanced about $17 \mathrm{~mm}$ from the bifurcation of the CCA until slight resistance was encountered (Fig. 1C). Transient ligation of the CCA was performed to interrupt the CCA blood flow and fixation of the occlusion guide wire (Fig. 1B). After 90 minutes occlusion time, we removed the transient ligation and withdrew the occlusion wire. The temporary clips were withdrawn, and all of the ICA and ECA blood flow recovered. The skin was sutured and the rats were allowed to wake up. Twenty four hours later we evaluated neurological deficit and sacrificed the rats. We did not use heparin at all during the procedure to make the MCAO.

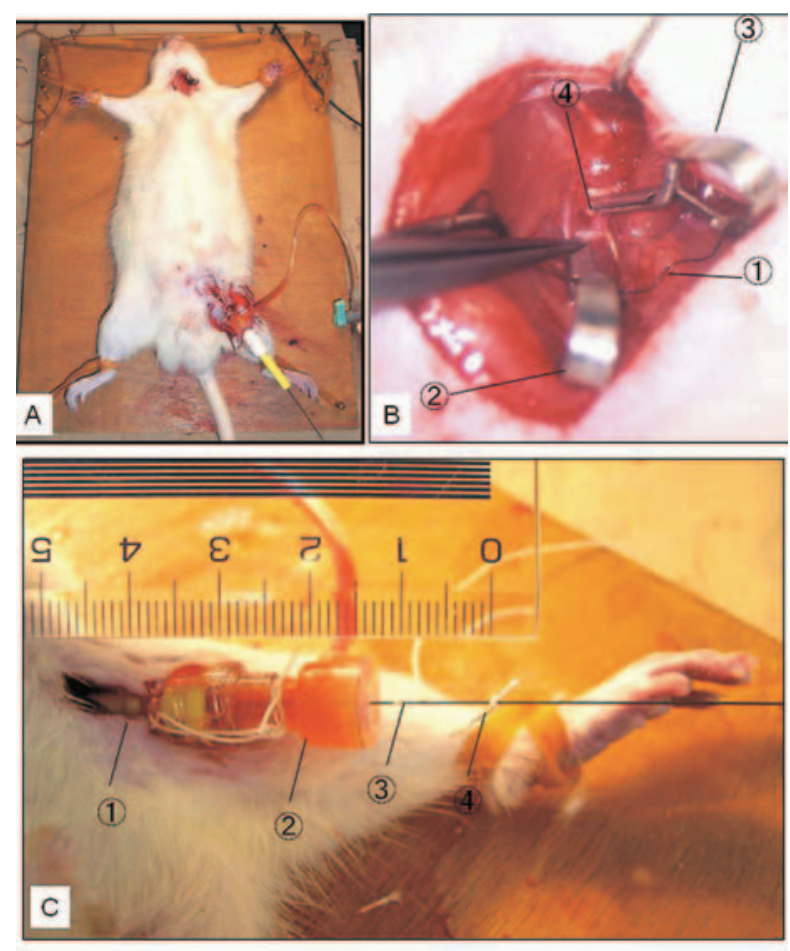

Figure 1 Intraoperative view

A: Overview of trans-femoral middle cerebral artery occlusion. B: Operative filed of left neck. (1) Transient ligation of common carotid artery. (2) Temporary occlusion clip on external carotid artery. (3) Temporary occlusion clip on pterygopalatine artery. (4) Occlusion micro guide wire. C: Cannulation of catheter. (1) Twenty-fourgage canula inserted into the left femoral artery. (2) Haemostatic valve. (3) 0.014 inch occlusion wire (Transend EX platinum tip). (4) Seventeen millimeter marker that prevents over-insertion of the wire. Numeric measure shows centimeters. 

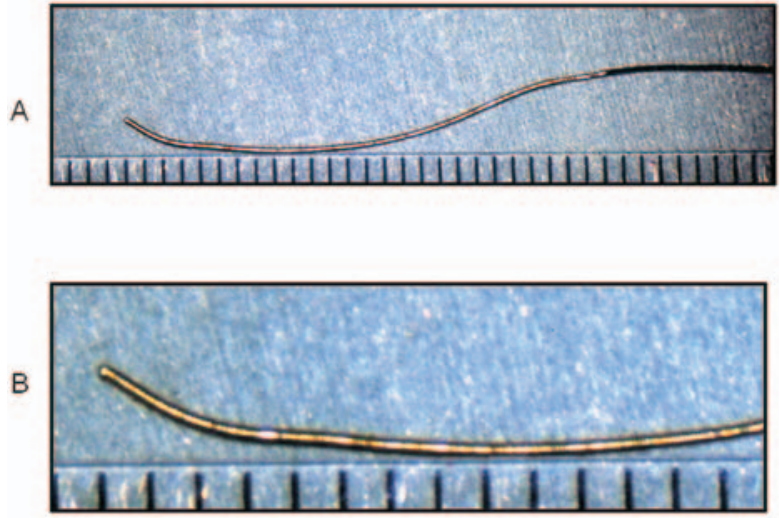

Figure 2 Occlusion micro guide wire.

A: Overview of the wire. The tip of the wire is slightly bent.

B: Magnification of wire. Tip of wire is round. Parallel marker shows 1 millimeter.

\section{Neurological Evaluation}

We carried out a neurological evaluation 24 $\mathrm{h}$ after the cerebral ischemia. Scoring was done blindly by MN on individual animals with a neurological score that was reported previously (Table 1$)^{7}$. The range of our neurological score was 1 to 15 and a value of 15 indicated no neurological deficit.

\section{Measurement of Infarct Volume}

The rats were decapitated under deep anesthesia. The brains were immediately removed and placed in ice-cold phosphatebuffered saline (PBS, Mitsubishi Chemical Medience Co. Tokyo, Japan) for 15 min. Brain tissue was cut coronally to a $2 \mathrm{~mm}$ thickness with a brain slicer (Harvard Apparatus; South Natick, MA). Brain slices were immersed in $2 \%$ 2,3,5-triphenyl - 2H - tetrazolium chloride (TTC, Kanto Chemical Co., Tokyo, Japan) solution at $37{ }^{\circ} \mathrm{C}$ for $5 \mathrm{~min}$, followed by $10 \%$ formaldehyde solution. The infarct area was traced and quantified by an image analysis system (Image J 1.33u).

\section{Statistical analysis}

Infarction volumes are expressed as mean
Table 1 Neurological Evaluation

\begin{tabular}{|c|c|c|c|c|}
\hline & \multicolumn{4}{|c|}{ Score } \\
\hline & 0 & 1 & 2 & 3 \\
\hline $\begin{array}{l}\begin{array}{l}\text { Spontaneous Activity } \\
\text { (in cage for } 3 \text { min) }\end{array}\end{array}$ & No movement & Barely moves & $\begin{array}{l}\text { Moves but does } \\
\text { not approach at } \\
\text { least } 3 \text { sides of } \\
\text { cage }\end{array}$ & $\begin{array}{c}\text { Moves and } \\
\text { approaches at } \\
\text { least } 3 \text { sides of } \\
\text { cage }\end{array}$ \\
\hline $\begin{array}{l}\text { Symmetry of } \\
\text { Movement (right } \\
\text { forelimb and hind } \\
\text { limb) }\end{array}$ & No movement & Slight movement & Moves slowly & $\begin{array}{c}\text { Moves } \\
\text { symmetrically }\end{array}$ \\
\hline Floor Walking & Falls down & Circling & $\begin{array}{l}\text { Inability to walk } \\
\text { straight }\end{array}$ & Normal walk \\
\hline $\begin{array}{l}\text { Beam Walking (3 cm } \\
\text { width) }\end{array}$ & Falls off & Hugs & Stands & Walks \\
\hline $\begin{array}{c}\text { Response to } \\
\text { Vibrissae touch on } \\
\text { rights side }\end{array}$ & & No response & Weakresponse & 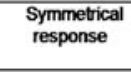 \\
\hline
\end{tabular}

\pm S.E.M. and neurological scores are expressed as median with I.Q.R. The correlation between infarction volume and neurological score was analyzed by JMP 8.01 (SAS institute Inc. Cary, $\mathrm{NC}$ ), and a p-value below 0.05 was accepted as statistically significant.

\section{Results}

One rat died before occlusion due to deep anesthesia. Two rats showed no neurological deficit or cerebral infarction. The success rate for producing infarction was $80 \%$ (Fig. 3A, B). No rat suffered subarachnoid hemorrhage and no rat died due to cerebral infarction.

The total infarction volume for this model was $218.6 \mathrm{~mm}^{3} \pm 28.5 \mathrm{se}$, infarction volume of basal ganglia was $94.4 \mathrm{~mm}^{3} \pm 9.4$ se, infarction volume of the cortex was $124.2 \mathrm{~mm}^{3} \pm 21.6 \mathrm{se}$. Median neurological score was $5.5(4.25$ - 7.75) $(\mathrm{n}=8)$.

The correlation between infarction volume and neurological score was statistically significant $(\mathrm{p}<0.05$, Fig. 3 C, D, E).

\section{Discussion}

We have developed a new rat MCAO model without sacrificing the ECA. This novel method is reproducible and reliable. Previously, all rat transient intraluminal suture MCAO models sacrificed the ECA with or without sacrificing the pterygopalatine artery. This sacrifice of the 

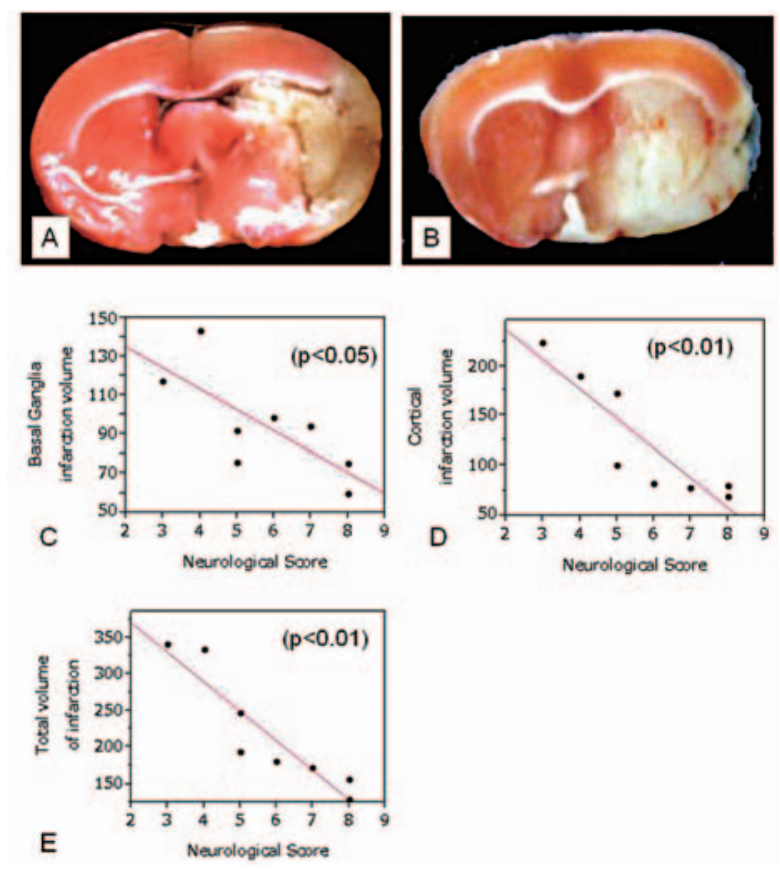

Figure 3 2,3,5-triphenyltetrazolium chloride hydrate staining and Correlation between infarction volume and neurological score.

A: Cerebral infarction with hemorrhagic change. B: Diffuse basal ganglia infarction occurred. C: Infarction volume of basal ganglia. D: Infarction volume of cerebral cortex. E: Total volume of infarction. Infarction volume correlated statistically significantly with the neurological score.

ECA induces ischemic tissue damage of the arterial territory, causing impaired mastication and swallowing ${ }^{6}$. We can avoid sacrificing the ECA via a trans-femoral approach to the ICA that is commonly used in neuro-interventional examination and treatment. Surgical approach to make the $\mathrm{MCAO}^{8,9)}$ also preserves the ECA, but such models require dissection of the temporal muscle, exposure of the cerebral cortex, and a change in intracranial pressure. Our new model does not require craniofacial surgical manipulation; this point is a major advantage.

The diameter of the 0.014 inch wire is 0.36 $\mathrm{mm}$ and the diameter of the end of the ICA is about $0.3 \mathrm{~mm}$, and the diameter of the entrance to the MCA and ACA is about $0.2 \mathrm{~mm}$. We can thus occlude the orifice of the MCA with 0.014 inch diameter guide wire. In a pilot study we used 0.016 inch diameter wire, but this wire could not reach the end of ICA safely due to the large caliber. Also, we tried a soft tip 0.014 inch diameter wire, but this very smooth guide wire easily perforated the end of the ICA or the orifice of the ACA. In this study we used a 0.014 inch diameter platinum tip guide wire with a $3 \mathrm{~cm}$ length grooved platinum coil tip. This grooved tip helps us maintain precise insertion of the occlusion wire during ligation of the CCA during occlusion. We required approximately 35 minutes from the incision of the neck to the beginning of MCA occlusion.

Rarely, the guide wire advanced to the left cardiac ventricle, causing arrhythmia, and we quickly withdrew the guide wire. If we use high resolution fluoroscopy, we will be able to approach the left ICA safely and quickly.

In the pilot experiment we used laser Doppler $\mathrm{CBF}$ monitoring, but in this study we did not use a $\mathrm{CBF}$ monitor to avoid complications and to maintain of the TF-MCAO advantage. Measuring $\mathrm{CBF}$ of the rat requires a craniotomy or translucent cranium. Either of these maneuvers can cause cortical injury. Intracranial pressure decreases due to a craniotomy, so CBF increases even with steady perfusion pressure. The invasiveness of the operation is increased if we add the skin incision for the $\mathrm{CBF}$ monitor. Additionally, dissection of the temporal muscle causes masticatory dysfunction that leads to an inadequate rat diet.

The success rate, volume of infarction, and neurological deficit were similar to our previous report ${ }^{4,5,7)}$. Infarction volume and neurological score also correlated statistical significantly, and this new TF-MCAO method is useful as a routine MCAO model. Moreover, we can guide the micro catheter to the ICA via the transfemoral artery by this method. Super selective drug delivery and infusion of neural stem cells ${ }^{10}$. 11) for a small animal can be done with this method. Our new method leads to a more useful 
model to study the basic neuroscience of rat ischemia.

\section{Acknowledgements}

This study was supported by a Hirosaki University Grant for Exploratory Research by Young Scientists. The authors have no financial interest in any of the drugs or materials described in this report. Micro guide wire was donated by Boston Scientific, Japan. This paper is already accepted by Journal of Neuroscience Methods.

\section{References}

1) Koizumi J YYNTOG. Experimental studies of ischemic brain edema 1 . A new experimental model of cerebral embolism in ratsin which recirculation can be introduced in the ischemia area. Jpn J Stroke 1986;8:1-8.

2) Garcia JH, Wagner S, Liu KF, Hu XJ. Neurological deficit and extent of neuronal necrosis attributable to middle cerebral artery occlusion in rats. Statistical validation. Stroke 1995;26:627-34.

3) Longa EZ, Weinstein PR, Carlson S, Cummins R. Reversible middle cerebral artery occlusion without craniectomy in rats. Stroke 1989;20:84-91.

4) Shimamura N, Matchett G, Yatsushige H, Calvert JW, Ohkuma H, Zhang J. Inhibition of integrin alphavbeta 3 ameliorates focal cerebral ischemic damage in the rat middle cerebral artery occlusion model. Stroke 2006;37:1902-9.
5) Shimamura N, Matchett G, Solaroglu I, Tsubokawa $\mathrm{T}$, Ohkuma $\mathrm{H}$, Zhang J. Inhibition of integrin alphavbeta3 reduces blood-brain barrier breakdown in focal ischemia in rats. J Neurosci Res 2006; 84:1837-47.

6) Dittmar M, Spruss T, Schuierer G, Horn M. External carotid artery territory ischemia impairs outcome in the endovascular filament model of middle cerebral artery occlusion in rats. Stroke 2003;34:2252-7.

7) Shimamura N, Matchett G, Tsubokawa T, Ohkuma $\mathrm{H}$, Zhang J. Comparison of silicon- coated nylon suture to plain nylon suture in the rat middle cerebral artery occlusion model. J Neurosci Methods 2006;156:161-5.

8) Buchan AM, Xue D, Slivka A. A new model of temporary focal neocortical ischemia in the rat. Stroke 1992;23:273-9.

9) Coyle P. Middle cerebral artery occlusion in the young rat. Stroke 1982;13:855-9.

10) Honma T, Honmou O, Iihoshi S, Harada K, Houkin $\mathrm{K}$, Hamada H, Kocsis JD. Intravenous infusion of immortalized human mesenchymal stem cells protects against injury in a cerebral ischemia model in adult rat. Exp Neurol 2006;199:56-66.

11) Kamiya N, Ueda M, Igarashi $H$, Nishiyama $Y$, Suda S, Inaba T, Katayama Y. Intra-arterial transplantation of bone marrow mononuclear cells immediately after reperfusion decreases brain injury after focal ischemia in rats. Life Sci 2008;83: 433-7. 